\title{
Tunable 0.7 conductance plateau in quantum dots
}

\author{
Yunchul Chung ${ }^{1}$, Sanghyun Jo², Dong-In Chang ${ }^{2}$, Hu-Jong \\ Lee $^{2} *$ M. Zaffalon ${ }^{3}$, V. Umansky ${ }^{3}$, and M. Heiblum ${ }^{3}$ \\ ${ }^{1}$ Department of Physics, Pusan National University, Busan 609-735, Republic of Korea \\ ${ }^{2}$ Department of Physics, Pohang University of Science and Technology, \\ Pohang 790-784, Republic of Korea \\ National Center for Nanomaterials Technology, \\ Pohang 790-784, Republic of Korea \\ ${ }^{3}$ Department of Physics, Weizmann Institute of Science, Rehovot 76100, Israel
}

(Dated: October 27, 2018)

\begin{abstract}
A new consistent approach to forming the 0.7 structure by using a quantum dot rather than a quantum point contact is demonstrated. With this new scheme, it was possible to tune on and off the 0.7 structure. The new 0.7 structure continuously evolved into a normal integer conductance plateau by varying the tuning condition. Unlike the conventional 0.7 plateau, the new 0.7 structure was observed even at low electron temperatures down to $100 \mathrm{mK}$, with unprecedented flatness. From our results, it is concluded that electron interference as well as the electron interaction effect should be taken into consideration to explain the 0.7 structure.

PACS numbers: 71.70.Ej, 73.23.Ad, 73.21.La
\end{abstract}

*Electronic address: hjlee@postech.ac.kr 
The quantization of conductance in units of $G_{0}\left(=2 e^{2} / h\right)$ through a quantum point contact (QPC) was first observed experimentally by two groups [1, 2]. The phenomenon is a result of adiabatic transmission of electrons through spin-degenerate non-interacting onedimensional (1D) channels. Shortly after the discovery, an additional plateau was observed at around $0.7 G_{0}$, which has been known as the 0.7 structure [3]. Many other experiments were followed to confirm the existence of this unusual plateau and to investigate its physical origin [4, 5, 6, 7, 8, 9, 10]. Since this anomalous conductance plateau cannot be explained in a single-particle picture, theoretical explanations based on electron-electron interaction, spin effect, and others have been suggested [11, 12, 13, 14, 15]. The spontaneous spin polarization model [11, 12] and the Kondo-related model [14] are most popular among them, which were supported experimentally by Thomas et al. [3] and Cronenwett et al. [7], respectively.

Nonetheless, no consensus has been reached about its origin yet and still contradicting experimental and theoretical results are reported. Furthermore, the 0.7 structure is not always revealed in a QPC although the phenomenon is regarded as intrinsic properties of 1D conduction channels. In most studies, however, the absence of the 0.7 structure was simply ignored and the condition for the occurrence of this structure has not been systematically investigated. We report a new approach to the formation of the anomalous 0.7 structure by using a quantum-dot structure, which allowed us to tune to the optimal observing condition at our disposal. This tunable 0.7 structure showed characteristics that were similar to the conventional ones in a QPC, but with additional new features. We believe our results will provide an insight into the nature of this ever-controversial phenomenon.

The quantum dots were fabricated on two-dimensional electron gas (2DEG) wafers based on a GaAs/AlGaAS heterostructure. The electron densities were around $2.5 \times 10^{11} \mathrm{~cm}^{-2}$ with the mobilities higher than $1.5 \times 10^{6} \mathrm{~cm}^{2} \mathrm{~V}^{-1} \mathrm{~s}^{-1}$ at $4.2 \mathrm{~K}$. The 2DEG layer resided 65 $\mathrm{nm}$ below the surface of the wafers. Two different types of quantum dots (Types A and B) were fabricated (Fig. 1). The electron temperature was estimated to be around 100 mK $(140 \mathrm{mK})$ for the Type-A (Type-B) sample. For convenience, all the gates were named individually as in Figs. 1(a) and 1(b). Conductance quantization behavior for individual QPC's was examined by applying negative voltages on the nose gate and one of the other three gates (QPC, side, and plunger gates) separately, while keeping the rest of the two gates at zero voltage. In each of these usual QPC geometries, only the normal integer conductance quantization was observed without any anomalies including the 0.7 structure. We believe 
the absence of anomalies was caused by the extreme narrowness (less than $50 \mathrm{~nm}$ in its width) of our gates. The zero-bias anomaly suggesting the Kondo character [7] was not observed, either, even at $100 \mathrm{mK}$, the base electron temperature used.

As in Fig. 1(c), the conductance anomaly started to appear only when certain negative voltages were applied to all the gates concurrently. To induce the conductance anomaly we first applied small fixed negative voltages to the QPC and the plunger gates, which were just strong enough to pinch the 2DEG under the gate. Only the nose gate was then varied to observe conductance quantization. For a fixed nose-gate voltage $V_{n g}$, the side-gate voltage $V_{s g}$ was scanned from -0.15 to $-0.6 \mathrm{~V}$ to tune on and off the conductance anomaly. Fig. 1(c) shows the conductance variation for the Type-B sample at the electron temperature of 140 $\mathrm{mK}$. The 0.7 structures are seen for $V_{s g}$ around $-0.46 \mathrm{~V}$ and $-0.34 \mathrm{~V}$. Thus, controlling $V_{s g}$ in the above circumstances provides a very convenient means to tune to the formation of the 0.7 structure. Higher-order anomalies like 1.7 and 2.7 structures, observed in ordinary QPC's, are also revealed. Similar features were obtained in the Type-A sample.

Figure 2(a) shows in detail how the 0.7 plateau for the Type-B sample evolves to and from the normal integer plateaus as $V_{s g}$ varies between $-0.24 \mathrm{~V}$ and $-0.41 \mathrm{~V}$. The 0.7 plateau becomes most pronounced for $V_{s g}=-0.34 \mathrm{~V}$ (the thick curve). For $V_{s g}=-0.24 \mathrm{~V}$ and above, however, no appreciable conductance anomalies were observed (see Fig. 1). The width of $G_{0}$ plateau reduces as a more negative voltage is applied to the side gate. For $V_{s g}=-0.34$ $\mathrm{V}$, it reduces to almost one half of that for $-0.24 \mathrm{~V}$, with the concurrent development of a clear plateau just below $0.8 G_{0}$. Further increasing the negative voltage to the side gate, the plateau reduces to a broad local maximum and transforms back to the $G_{0}$ plateau for $V_{s g}=-0.41 \mathrm{~V}$. As shown in Fig. 1(c) these generic processes repeat for varying $V_{s g}$. The width of the $G_{0}$ plateau changes noticeably with $V_{s g}$ varying from $-0.24 \mathrm{~V}$ to $-0.41 \mathrm{~V}$, while the pinch-off voltage remains almost unaltered. This implies that the recurrent change of the width of the normal integer plateaus for varying $V_{s g}$ was not caused by the possible electrostatic coupling between the side and the QPC gates.

Figure 2(b) shows the modulation of the width of the $G_{0}$ plateau for the Type-B sample as a function of $V_{s g}$. The strong modulations are shown for $V_{s g}$ lower than $-0.3 \mathrm{~V}$, along with clear 0.7 plateaus (two front arrows). At $V_{s g}=-0.22 \mathrm{~V}$ a faint 0.7-like structure (the rear arrow) is also evident. Both samples show well-formed plateaus for the conductance just below $0.8 G_{0}$. For the Type-A sample, with the average spacing between the side and 
the QPC gates closer than in the Type-B sample, the plateau is substantially flatter. Figure 2(c) shows the flatness of the 0.7 structure of the Type-A sample. The average conductance for $V_{n g}$ between $-1.120 \mathrm{~V}$ and $-1.134 \mathrm{~V}$ is $0.791 G_{0}$, with the conductance deviation less than $0.1 \%$ of the averaged value. No 0.7 structures reported to date have the flatness as high as this. It is highly unlikely that such high flatness is induced by the suppression of the Kondo resonance peak [14]. The flatness can hardly be explained without introducing an energy gap in the model. Along this line, the spin-gap model [11, 12] may be the best candidate to interpret the data. It, however, does not explain the repeated occurrence of the 0.7 structure for varying $V_{s g}$. According to the model, the spin gap widens monotonically with increasing the electron carrier density in a QPC. The electron carrier density inside our QPC gate supposedly decreased monotonically for more negative $V_{s g}$, the trend of which was in contradiction to the clear occurrence of the 0.7 structure.

In this study the electron carriers were intended to be weakly localized inside the quantum dot formed by the carrier-confining gates, mimicking the Kondo [16] configuration that was claimed to cause the 0.7 structure [14]. The side-gate voltages in the presence of the 0.7 structure [denoted by two front arrows in Fig. 2(b)], $V_{s g}=-0.34 \mathrm{~V}$ and $-0.46 \mathrm{~V}$, corresponded to opening of 5.9 and 3.1 conducting channels between the nose and the side gates, respectively. Thus, the carriers were not effectively confined inside the quantum dot even in the presence of the 0.7 structure. A weak 0.7 structure [denoted by the rear arrow in Fig. 2(b)] was also observed even for much less confining side-gate voltage, $V_{s g}=-0.22 \mathrm{~V}$, which corresponded to opening of more than 100 conducting channels. This analysis, thus, excludes the possibility of the Kondo origin of the 0.7 structure at least in our sample configuration.

The temperature dependence of the 0.7 structure of the Type-A sample is shown in Fig. 3. Before varying the temperature, the side gate was set at the optimum voltage $\left(V_{s g}=-0.19 \mathrm{~V}\right)$ for the formation of the 0.7 structure at the base electron temperature. As shown in Fig. 3(a) the tunable 0.7 plateau gets weaker as the temperature increases and eventually vanishes for $T=800 \mathrm{mK}$. The temperature dependence of the conductance in Fig. 3(b), similar to the one in Fig. 3(a) but taken for a slightly more negative sidegate voltage $\left(V_{s g}=-0.20 \mathrm{~V}\right)$, reveals a local conductance maximum rather than a plateau at the base electron temperature. As the temperature increases the local maximum gets smeared and gradually transforms into the 0.7 structure, forming a perfect plateau at the temperature around $520 \mathrm{mK}$. This temperature-dependent evolution of the plateau is similar 
to the feature of the regular 0.7 structure observed in the conventional QPC's [3, 7], where it has been known that the 0.7 structure develops only at relatively high temperatures around $1 \mathrm{~K}$.

The source-drain bias dependence of the conductance at different $V_{n g}$ was examined with $V_{s g}$ set to show an almost normal integer conductance plateau. As seen in Fig. 4(a), the measured conductance curves around zero bias are somewhat asymmetric, due to the imbalance of the potential build-up around each gate. The 0.7 structures are seen for the bias around $0.87 \mathrm{mV}, 0.46 \mathrm{mV},-0.36 \mathrm{mV}$, and $-0.87 \mathrm{mV}$. One also notices that the 0.7 structure is a little clearer in negative biases, which correspond to the situation where electrons were injected to the side gate first. Since the side gate was more open than the QPC gate, the carrier density in this case was higher in the quantum-dot region between the side and the QPC gates than the case of electrons injected to the QPC gate first. If the new 0.7 structure observed in our samples was caused by some correlation of electrons in the carrier-confining quantum-dot region, a stronger effect was more likely to be observed when the electron concentration was higher as in this study.

Measurements similar to the ones leading to Fig. 1(c) were repeated in a magnetic field of $0.15 \mathrm{~T}$ applied perpendicular to the plane of the 2DEG. As shown in Fig. 4(b), in this relatively low magnetic field, the 0.7 structures were almost entirely suppressed, leaving only normal integer conductance plateaus. A very weak field like $0.05 \mathrm{~T}$ was high enough to considerably weaken the 0.7 structures. No such behavior has been reported in the conventional 0.7 conductance anomaly [17].

With two carrier-confining gates (the side and the QPC) arranged in series in the direction of electron transport, the interference may have taken place for the electrons moving back and forth between the gates. It thus can be inferred that such an interference may have caused the 0.7 plateau in our measurements. In Fig. 4(c), the conductance is plotted as a function of $V_{s g}$, with the nose gate fixed around the middle of the 0.7 plateau. A clear interference pattern is revealed as $V_{s g}$ varies. Marked by arrows in Fig. 4(c) are the positions of $V_{s g}$ where the 0.7 structures were observed. Although the interference is evident, not much correlation exists between the 0.7 structure and the interference pattern. The 0.7 structure formed at $V_{s g}=-0.22 \mathrm{~V}$ locates at the local conductance maximum, while those formed for $V_{s g}$ at $-0.46 \mathrm{~V}$ and -0.34 locate somewhat away from the local conductance maxima. This implies that the 0.7 structure was not caused by the interference only. It is possible that 
multiple transmission modes participated in the interference. If that was the case no direct correlation could exist between the 0.7 structure and the interference pattern as in Fig. 4(c).

The extreme flatness of the 0.7 structure over a wide range of $V_{n g}$ as in Fig. 2(c) is not explained by the simple electron interference effect, either. Varying the $V_{n g}$, which is bound to alter the phase of the interfering electrons, should have resulted in the continuous change in the conductance as a function of $V_{n g}$. In addition, the observation of the 0.7 plateau at high temperatures shown in Fig. 3(b) is hardly explained by the interference. At such high temperatures the interference is supposed to be much reduced due to the decoherence of electron phases. On the other hand, the repetition of 0.7 structure as a function of side-gate and bias voltages resembles the feature often seen in the electron interference effect.

Recently, it has been reported that a 0.5 plateau can be realized by changing the potential landscape of $1 \mathrm{D}$ wire by using a scanning probe tip [18]. The 0.5 structure was observed when the potential away from the main confinement potential landscape was perturbed. In this case, electrons scattering back and forth between the main and perturbed potential possibly resulted in the interference. Even in conventional QPC's the electron interference is likely to happen since the shape of the quasi-1D wire formed by QPC gates is usually distorted by the formation of the unintended impurity potential. An exception takes place for an extremely short QPC formed by narrow gates. In this study, no conductance anomaly was observed for QPC's, the widths of which were shorter than $50 \mathrm{~nm}$. We believe that the 0.7 structure has not been observed consistently in ordinary QPC's due to the varying distortion of the potential landscape from sample to sample. The difference in features of the 0.7 structure between our samples and conventional QPC's can be a result of the difference in the detailed shape of the potential landscape. Although we consider the electron interference is the main causes of the 0.7 anomaly, all of its features are not explicable in terms of the electron interference only. Thus, both the interference and the interaction effect are believed to play essential roles in causing the 0.7 anomaly.

In summary, we have demonstrated a new approach to forming the 0.7 plateau in tunable quantum-dot structures. In clear contrast to the conventional one in an ordinary QPC structure, the 0.7 plateau in our study was observed even at very low electron temperatures with unprecedented flatness. This new 0.7 plateau evolved into a normal integer plateau when the negative side-gate voltage was varied. Since the 0.7 plateau occurs even when carriers are not fully confined in the quantum-dot region the Kondo mechanism is ruled out 
for the cause of the anomaly at least in our tunable quantum-dot structures. Interference as well as enhanced interaction among electrons in and around a QPC is believed to be the main cause of the anomaly. This new approach allows one to tune to the formation condition of the 0.7 plateau at one's disposal. The tuning, however, turns out to be somewhat subtle, which may explain the reason the conventional 0.7 structure is not always observed in ordinary QPC's.

This work was supported by Electron Spin Science Center, in Pohang University of Science and Technology, administered by KOSEF, and by the Korea Research Foundation Grant, KRF-2005-070-C00055 and KRF-2004-003C00076. 


\section{REFERENCES}

[1] B. J. van Wees et al., Phys Rev. Lett, 60, 848 (1988).

[2] D. A. Wharam et al., J. Phys. C 21, L209 (1988).

[3] K. J. Thomas et al., Phys. Rev. Lett. 77, 135 (1996).

[4] K. J. Thomas et al., Phys. Rev. B 61, R13365 (2000).

[5] C.-T. Liang et al., Phys. Rev. B 61, 9952 (2000).

[6] A. C. Graham et al., Phys. Rev. B 72, 193305 (2005).

[7] S. M. Cronenwett et al., Phys. Rev. Lett. 88, 226805 (2002).

[8] L. P. Rokhinson, L. N. Pfeiffer, and K. W. West, Phys. Rev. Lett. 96, 156602 (2006).

[9] P. Roche et al., Phys. Rev. Lett. 93, 116602 (2004).

[10] R. de Picciotto et al., Phys. Rev. Lett. 92, 036805 (2004).

[11] D. J. Reilly et al., Phys. Rev. Lett. 89, 246801 (2002).

[12] P. Havu et al., Phys. Rev. B 70, 233308 (2004).

[13] K.-F. Berggren and I. I. Yakimenko, Phys. Rev. B 66, 085323 (2002).

[14] Y. Meir, K. Hirose, and N. S. Wingreen, Phys. Rev. Lett. 89, 196802 (2002).

[15] D. Meidan and Y. Oreg, Phys. Rev. B. 72, 121312(R) (2005).

[16] D. Goldhaber-Gordon et al., Nature 391, 156 (1998).

[17] The 0.7 structure was also examined for fields applied in parallel with the 2DEG. The transverse magnetoresistance measurements could not be made, however, because the perpendicular field component caused by any possible field misalignment from the planar direction quickly suppressed the 0.7 structure.

[18] R. Crook et al., Science 312, 1359 (2006). 


\section{FIGURE CAPTIONS}

Figure 1. SEM pictures of two different types of quantum dots; (a) Type A and (b) Type B. (c) The conductance measured for the Type-B sample, with the QPC-gate and the plunger-gate voltages fixed at $-0.7 \mathrm{~V}$ and $-0.23 \mathrm{~V}$, respectively, as a function of nose-gate voltages $V_{n g}$ while varying the side-gate voltages $V_{s g}$ from $-0.15 \mathrm{~V}$ to $-0.6 \mathrm{~V}$ at intervals of $0.01 \mathrm{~V}$. The 0.7 structure is present at $V_{s g}=-0.46 \mathrm{~V}$ and $-0.34 \mathrm{~V}$ (lower solid curves). Weak 0.7 anomaly is also seen at $V_{s g}=-0.22 \mathrm{~V}$ (upper solid curve). Data for $V_{s g}$ from $-0.24 \mathrm{~V}$ to $-0.41 \mathrm{~V}$ are shown in more detail in Figure 2.

Figure 2. (color) (a) The evolution of the 0.7 plateau of the Type-B sample to and from the normal integer plateaus for varying $V_{s g}$ from $-0.24 \mathrm{~V}$ to $-0.41 \mathrm{~V}$ at intervals of $0.01 \mathrm{~V}$. The thick curve is the conductance taken for $-0.34 \mathrm{~V}$. (b) The conductance as a function of $V_{s g}$ and $V_{n g}$ put in a 3D-pseudo-color plot. The positions of the 0.7 structure are marked by arrows for clarity. (c) The conductance as a function of the $V_{n g}$ for the Type-A sample at the electron temperature of $100 \mathrm{mK}$, with the side and the QPC gates were set at -0.19 $\mathrm{V}$ and $-0.85 \mathrm{~V}$, respectively.

Figure 3. (color online) The temperature dependence of 0.7 structure for the Type-A sample. The nose and the QPC gates were set to show (a) the 0.7 structure and (b) a resonance-like local conductance maximum rather than a plateau at $92 \mathrm{mK}$. The fridge temperatures were $92,152,250,365,420,520,630,730$, and $830 \mathrm{mK}$ from bottom to top [without the data for $630 \mathrm{mK}$ in (b)].

Figure 4. (a) The differential conductance of the Type-B sample, measured as a function of the source-drain bias voltage for varying $V_{n g}$ from $-632 \mathrm{mV}$ to $-1061 \mathrm{mV}$ at intervals of $3 \mathrm{mV}$. The QPC and the plunger gates were set at $-0.7 \mathrm{~V}$ and $-0.23 \mathrm{~V}$, respectively, while the side gate was at $-0.39 \mathrm{~V}$. (b) The tunable 0.7 structure taken in the same measurement condition as the data in Fig. 1(c) but in a perpendicular magnetic field of $0.15 \mathrm{~T}$. (c) The 
conductance as a function of $V_{s g}$ with $V_{n g}$ set at $-0.964 \mathrm{~V}$. The 0.7 structures were observed at $V_{s g}$ marked by the arrows. 


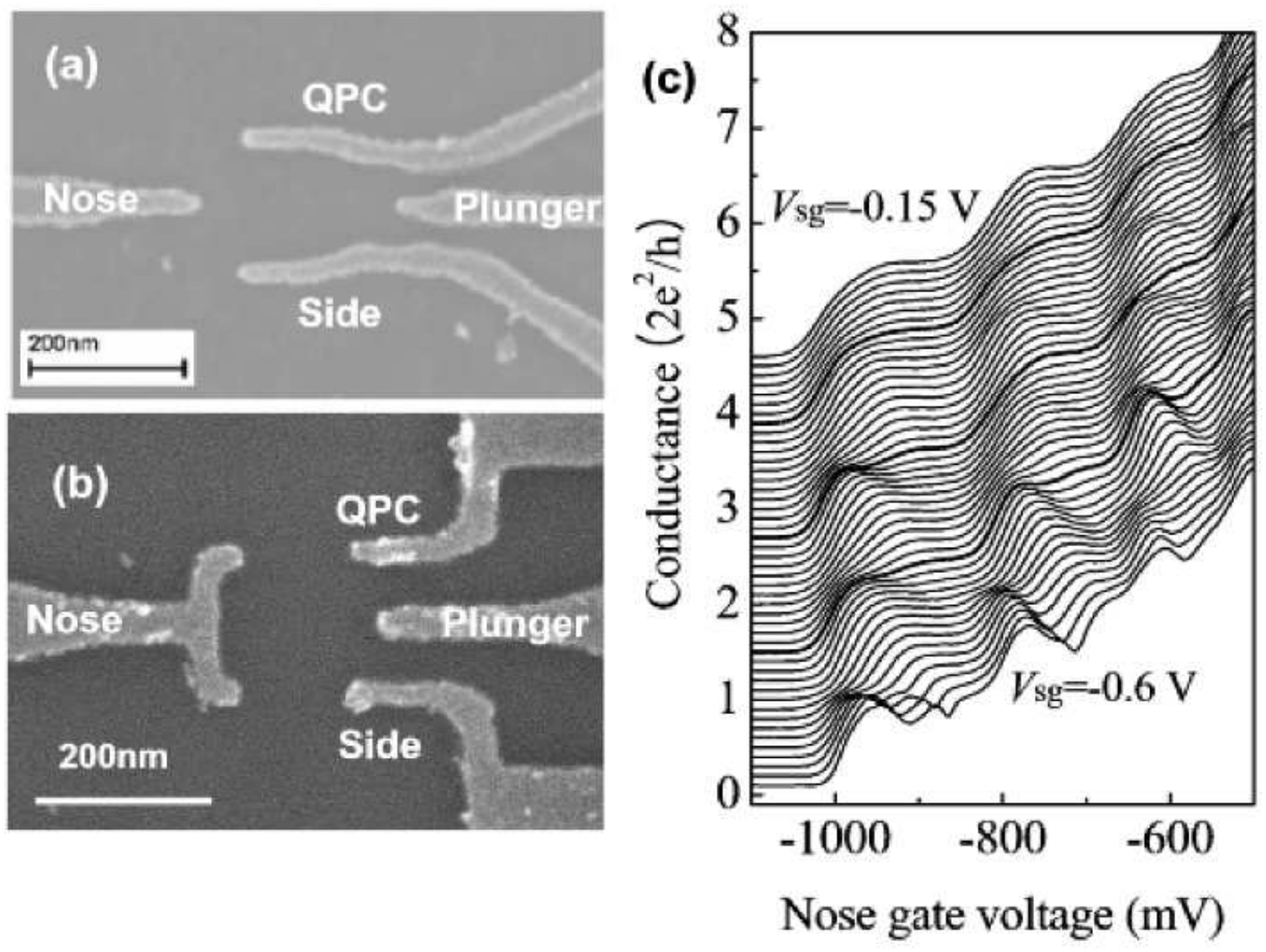

FIG. 1: 

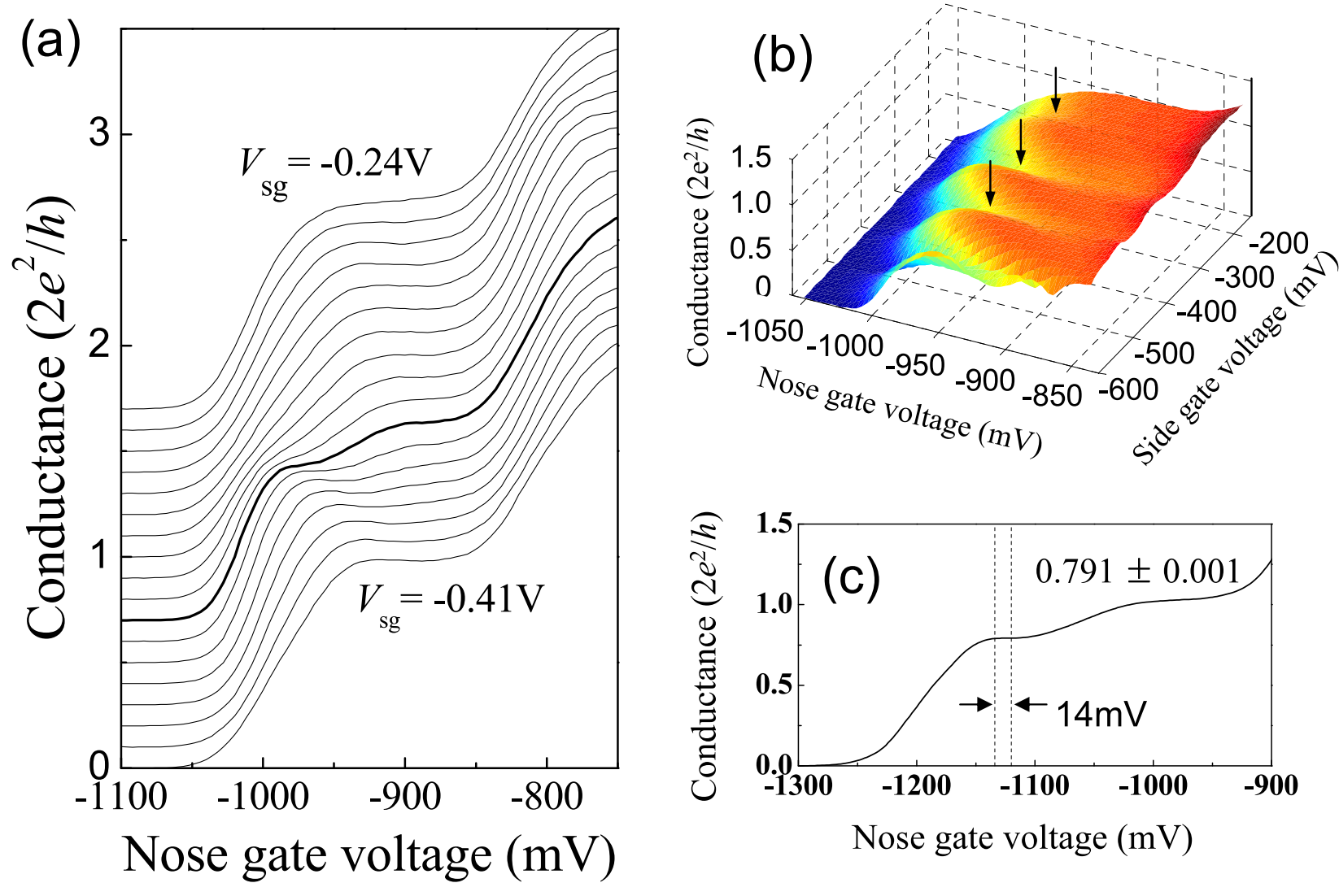

FIG. 2: 

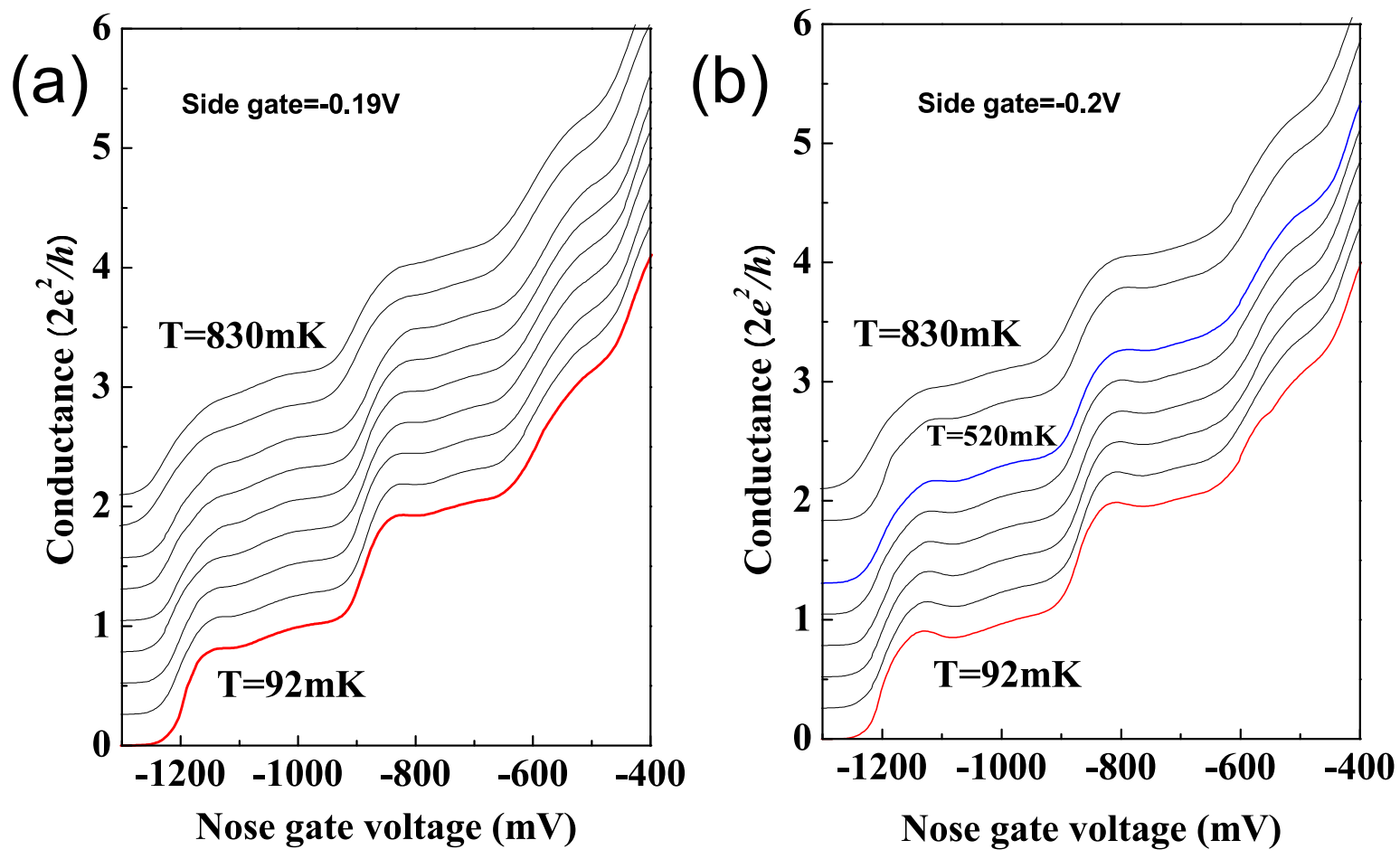

FIG. 3: 

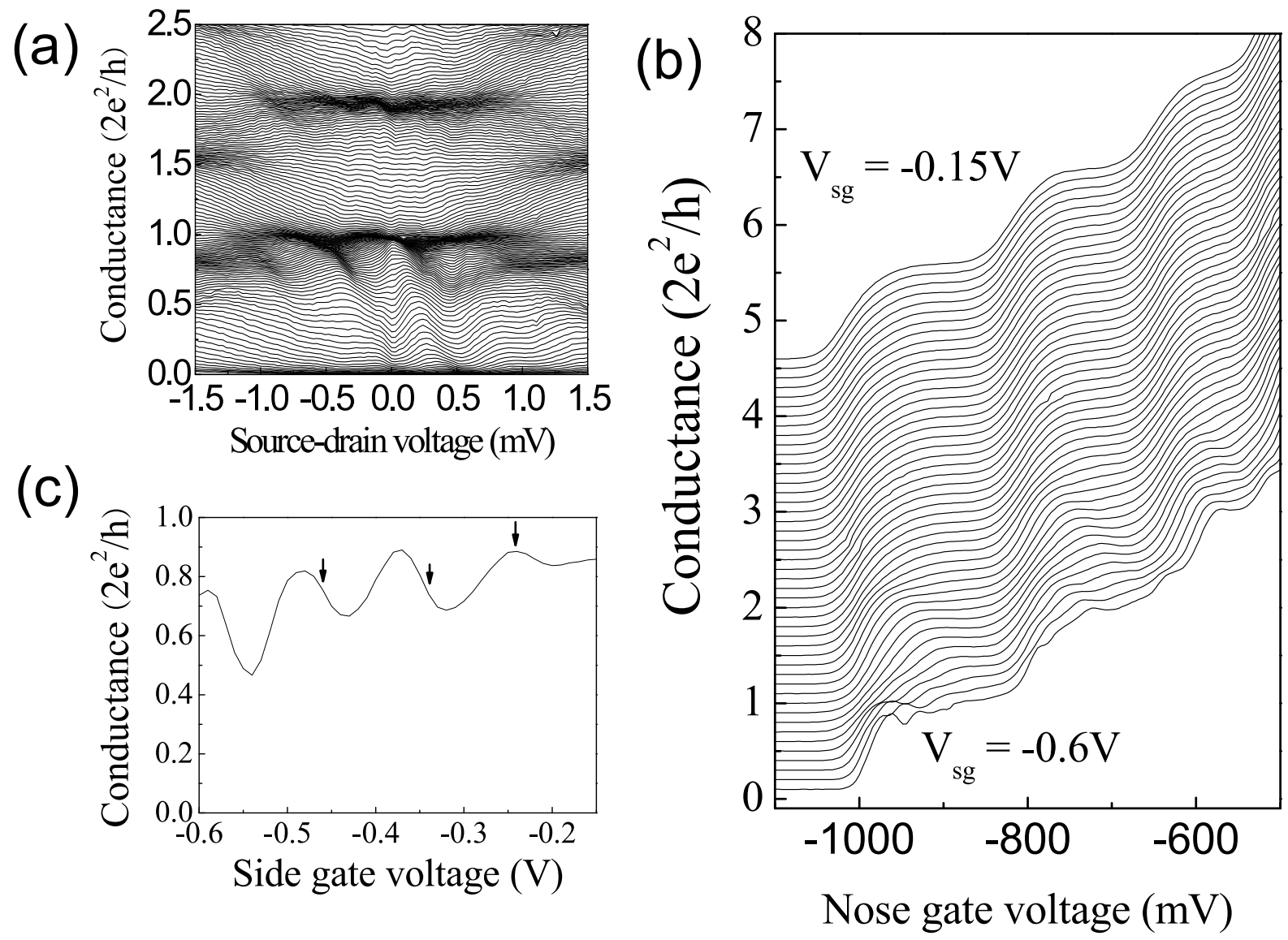

FIG. 4: 